\title{
A low complexity partial transmit sequence scheme by use of dummy signals for PAPR reduction in OFDM systems
}

\begin{abstract}
In this paper a novel technique for reducing the peak to average power ratio (PAPR) in OFDM systems by using the combination of the dummy sequence insertion (DSI) and partial transmit sequence (PTS) is proposed. In DSI increasing the number of dummy sequence decrease the transmission efficiency (TE) and in PTS the complexity increases when the number of subblock increases. Unlike the conventional PTS which needs several inverse fast fourier transform (IFFT) operations, the proposed DSI-PTS technique requires half IFFT operations only, while the PAPR performance is even better. So, it can remarkably reduce the computational complexity. Simulation results are examined with IEEE 802.16-2004 standard. By applying the DSI-PTS method about $0.5 \mathrm{~dB}$ reduction in PAPR at complementary cumulative distribution function $(\mathrm{CCDF})$ of $0.01 \%$ is achieved compared to the conventional PTS while the complexity is reduced.
\end{abstract}

Keyword: PAPR; OFDM; DSI; PTS; WiMAX 